\title{
ESSENTIAL PTX3 BIOLOGY (NOT ONLY) FOR CARDIOLOGISTS AND CARDIAC SURGEONS
}

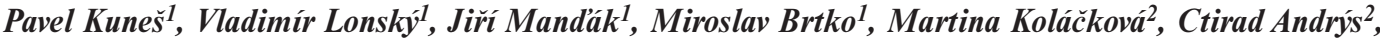 \\ Manuela Kudlová ${ }^{2}$, Jan Krejsek ${ }^{2}$ \\ Charles University in Prague, Faculty of Medicine in Hradec Králové and University Hospital Hradec Králové, \\ Czech Republic: Department of Cardiac Surgery ${ }^{1}$, Institute of Clinical Immunology and Allergology ${ }^{2}$
}

Summary: Inflammation has been recognized to form an integral part of the atherosclerotic process. Much consideration has been given lately to the role played in atherogenesis by C-reactive protein (CRP). Although not accepted unequivocally, CRP appears to be not only a marker, but also an active mediator of the atherosclerotic process. Pentraxin 3 (PTX3) is a newly identified acute phase reactant which shares some structural and some functional properties with CRP. On the other hand, pentraxin 3 displays unique biological properties of its own, including a possible role in the pathogenesis of cardiovascular diseases and in processes accompanying the natural evolution of surgical wounds. This review article discusses recent information concerning basic pentraxin 3 biology in inflammation and in innate immunity reactions as viewed by a cardiologist in the context of acute coronary events and by a surgeon in patients struck with multiple wounds who are at the same time menaced by bacterial infections.

Key words: Pentraxin 3; C-reactive protein; Acute phase response; Apoptosis; Macrophages; Dendritic cells; Innate immunity

\section{Introduction}

Two so-called short pentraxins, C-reactive protein (CRP) and the serum amyloid P component (SAP), are known to operate as acute phase reactants. In humans, CRP is beyond any doubt the more important of the two, while the function of SAP is only partially understood. In mice on the other hand the reverse situation is encountered, with CRP playing negligible, if any, role in the acute phase response. Both pentraxins are produced in the liver, with interleukin (IL)-6 being the key regulatory mediator, at least as far as human CRP is concerned. Additional sites of CRP production have been identified in the last few years, e.g. the kidney and the vascular wall. However, the respective contribution of these alternative sources to the overall CRP elevation during an acute inflammation does not seem to attain much biological significance (5). By contrast, the role of CRP as a marker or even a mediator of lowgrade chronic inflammatory processes, such as rheumatoid arthritis or atherosclerosis, has been a subject of much experimental and clinical research. Although not accepted unequivocally by the scientific community, adherents of CRP's active role in these pathological processes markedly predominate.

To make the issue of atherosclerosis as an inflammatory disease more complicated, a recently identified long pentraxin PTX3 has entered the scene. All pentraxins belong among very old proteins. Vertically, throughout evolution, their composition has been conserved with only insignificant modifications. Horizontally, among diverse species, their presence ranges from the horseshoe crab Limulus polyphaemus up to man (38). Pentraxin 3 was originally cloned as an interleukin (IL)-1 $\beta$-inducible gene in endothelial cells (9) or as a tumor necrosis factor (TNF)- $\alpha$-inducible gene (alternatively named TSG14) in fibroblasts (25). Pentraxin 3 consists of a C-terminal pentraxin-like domain of 203 amino acids which is common to other pentraxins, coupled to an additional, structurally unique $\mathrm{N}$-terminal portion made up of 178 amino acid residues. Pentraxin 3 protomers assemble into higher order complexes formed by 10-20 monomer units (i.e. dekamers or dodekamers) (7). Unlike CRP, which is manufactured predominantly in the liver, pentraxin 3 is produced directly at sites of tissue damage, with the liver playing only a marginal role in its genesis. Pentraxin 3 production occurs additionally at sites remote from the injured tissue, notably in the vascular endothelium of skeletal muscles and the heart (19). Pentraxin 3 synthesis is induced by proinflammatory cytokines TNF- $\alpha$ or IL- $1 \beta$, and by microbial wall constituents such as the lipopolysaccharide (LPS), mycobacterial liparabinomannans and the outer membrane protein A (OmpA) of Gram-negative bacteria (37). All these moieties of microbial origin employ the toll-like receptor (TLR) signaling pathway in order to transmit their respective biological signals into the target cells (8). Interferon (IFN)- $\gamma$, which in most cases acts synergistically with LPS, inhibits pentraxin 3 production in mono- 
cytic cell lines. By contrast, interleukin-10, the most potent anti-inflammatory cytokine as yet recognized, synergizes with LPS in the production of pentraxin 3 (12). IL-6, the major cytokine governing CRP synthesis in the liver, plays a negligible role in pentraxin 3 production, being limited to rare cases of Kaposi's sarcoma or Castleman's disease $(22,26)$.

\section{Pentraxin 3: possible implications in coronary artery disease}

The occurrence of pentraxin 3 is predominantly observed in the vascular wall. In patients with small vessel vasculitides, pentraxin 3 is found in deposits formed by dying leukocytes that assemble in perivascular areas of the diseased vessels or even systemically in the circulation, where it is supposed to reflect the overall endothelial activation (14). Pentraxin 3 synthesis, both mRNA and protein, can be induced by atherogenic lipoproteins in vascular smooth muscle cells (23). In human endothelial cells, pentraxin 3 supports the synthesis of tissue factor which has been induced by TNF- $\alpha$, IL-1 $\beta$ or lipopolysaccharide, all of them concomitant inducers of pentraxin 3 production (28). In atherosclerotic plaques, pentraxin 3 is produced by infiltrating macrophages and by scattered endothelial cells (34). Peri and colleagues found in their cohort of 37 patients with acute myocardial infarction (AMI) that plasma levels of PTX3 peaked about seven hours after the onset of chest pain. In AMI patients, plasma levels of CRP are known to increase both as a part of the acute phase response and as a reflection of the damaged myocardium. Peak levels of CRP, however, can be assessed only later, about 24 to 48 hours after the initial AMI symptoms. Peak levels of plasma pentraxin 3 attained by AMI patients were shown to occur independently of the extent of myocardial tissue necrosis or of the gravity of incident heart failure assessed by the Killip class at entry. Consequently, in the setting of acute myocardial infarction pentraxin 3 presents the potential advantage of an early marker of heart muscle damage irrespective of its extent, whose peak plasma levels are available well ahead of the elevation of plasma CRP (31). The same research team showed in addition that pentraxin 3 was a marker which supplied the best prognostic information concerning three month mortality after the index event. Among their 724 AMI patients, those whose first day plasma levels of pentraxin 3 exceeded $10.73 \mathrm{ng} / \mathrm{ml}$, exhibited a three-fold higher risk of dying within the next 3 months compared to patients whose pentraxin 3 levels did not reach $5.49 \mathrm{ng} / \mathrm{ml}$ at the same point in time. The prognostic information conferred by pentraxin 3 was superior in accuracy to prognostic values mirrored by levels of creatine kinase, troponin $\mathrm{T}$ or $\mathrm{N}$-terminal pro-brain natriuretic peptide, not to mention CRP itself (24).

\section{Pentraxin 3 and the complement system}

Pentraxin 3 exhibits a high-affinity binding to the globular head of C1q, the first component of the complement cas- cade. This binding displays the quality of a competitive inhibition. In an environment with excess CRP or SAP, the long pentraxin is displaced from its complex with $\mathrm{Clq}$ by the short pentraxin competitors, which bind to $\mathrm{C} 1 \mathrm{q}$ with a comparable affinity. The reverse takes place if CRP or SAP is bound to $\mathrm{C} 1 \mathrm{q}$ in the presence of excess pentraxin 3. Once bound to $\mathrm{C} 1 \mathrm{q}$, pentraxin 3 either initiates or inhibits activation of the complement cascade via the classical pathway. The respective effect will depend on the actual environmental conditions. Locally formed pentraxin 3 activates complement which is available within inflamed or injured tissues, thereby supporting local defense reactions. Pentraxin 3 present in the circulation, i.e. remote from the site(s) of local inflammation, inhibits activation of complement in that pentraxin 3 molecule inserts between the C1q globular head and the following components of the complement cascade. Due to occupancy by pentraxin 3 of their respective binding sites, these complement components are hindered from direct contact with $\mathrm{Clq}$ which in turn is necessary for the activation process to continue. In this position, pentraxin 3 acts counter to an overwhelming activation of the complement cascade during systemic inflammatory processes (29).

Pentraxin 3 is endowed with the quality of an opsonin, which acts in concert with the complement fragment $\mathrm{C} 3 \mathrm{~b} /$ $\mathrm{iC} 3 \mathrm{~b}$ on the surface of apoptotic cells. This activity of pentraxin 3 supports removal of apoptotic cells and tissue debris, which in itself is a prerequisite for proper wound healing (36). In its role of an opsonin, pentraxin 3 targets some micro-organisms, above all the conditionally pathogenic fungus Aspergillus fumigatus. If sufficiently opsonized by pentraxin 3 , aspergillic conidia are ingested by the host's alveolar macrophages and subsequently cleared from the lungs (16). Furthermore, Diniz and colleagues showed that recombinant pentraxin 3 binds to zymosan and Paracoccidioides brasiliensis, thereby increasing phagocytic ingestion of the pentraxin 3-covered particles by peritoneal macrophages of wild-type mice. This finding is in keeping with the importance of the opsonic role of pentraxin 3 in defense reactions and implies the existence of a pentraxin 3-specific receptor on target cells. This receptor, however, has not yet been identified (11).

\section{Pentraxin 3 and KpOmpA}

Apart from the C1q globular head of complement, pentraxin 3 further binds to:

1. the fibroblast growth factor-2 (FGF2), a mediator implicated in angiogenesis,

2. the extracellular matrix protein TSG-6, which supports tissue repair/remodeling,

3. the outer membrane protein A from Klebsiella pneumoniae (KpOmpA), a newly recognized mediator of inflammation.

Recognition of KpOmpA by the host is mediated via scavenger receptors LOX-1 (lectin-like oxidized low-density lipoprotein receptor 1) and SREC-I (scavenger receptor expressed by endothelial cell-I). Both receptors are expressed 
on macrophages and dendritic cells (DCs). Activation by KpOmpA of the host's innate immunity effector cells, primarily immature DCs, is accomplished via toll-like receptor (TLR)-2. Transmembrane signaling in immature DCs, which is set off by KpOmpA, up-regulates costimulatory molecules CD40, CD80 (B7-1), CD86 (B7-2), intercellular adhesion molecule ICAM-1 (CD54), and MHC class I or class II molecules (CD for cluster of differentiation). KpOmpA also induces production by dendritic cells of IL-12 and TNF- $\alpha$. Dendritic cell maturation is thus next to complete. Last but not least, KpOmpA targets exogenous antigens to the MHC class I presentation pathway. All the above mentioned activities of KpOmpA culminate in mobilization of potent antibacterial immune reactions. Invading bacteria are eliminated by macrophages in conjunction with the complement system and the pentraxins. Antigen-specific immune responses, initiated by maturing DCs, are executed by cytotoxic CD8+ T-lymphocytes. In this respect, dendritic cells present a transition from innate to acquired immunity responses. Exogenous antigens, which have been endocytosed by DCs via KpOmpA-binding elements, gain access to the cytosolic MHC class I presentation pathway. Effector/ cytotoxic CD8+ T lymphocytes can thus be generated even in the absence of CD4+ T lymphocyte help (21). Cellular signaling exerted by KpOmpA furthermore leads to the production by monocytes/macrophages and by dendritic cells of pentraxin 3. This feature of $\mathrm{KpOmpA}$, which itself is an archetypical pathogen-associated molecular pattern, reflects the seemingly ambiguous or even contradictory nature of the innate immunity reactions, which at the same time combat infection and set the stage for the termination of inflammatory processes, the latter being a prerequisite for repair and regeneration of wounded tissues. Pentraxin 3, along with the complement system, fits in with both of these ambiguous activities. It is an effective mediator of the humoral arm of innate immunity and at the same time it supports elimination of the host's own damaged cells, while meticulously sparing those that have survived cytotoxic attack unleashed by the defense reactions (20).

\section{Pentraxin 3 and apoptotic cells}

All pentraxins bind to dying cells, whose number rapidly increases in inflamed or injured tissues, in order for the host to get rid of, by phagocytosis, cellular corpses and intracellular components released by injured or dying cells. Clearance of apoptotic cells and subcellular constituents thereof by macrophages, in conjunction with the pentraxins, starts off paracrine release of anti-inflammatory mediators such TGF- $\beta$ (transforming growth factor- $\beta$ ), which guarantee an immunologically silent cellular death (13). Should this homeostatic mechanism be perturbed, highly immunogenic peptides, which in normal circumstances are integrated within the host's live cells, would inevitably enter the extracellular milieu. Once beyond the protective line of the outer cellular membrane, intracellular components of the host's tissues are identified by his or her DCs as foreign antigens. If not removed out of reach of this keen surveillance machinery with ample advance, catastrophic auto-immune reactions, provoked by the host's own intracellular antigens, would set in without any restraint (2). In promoting timely elimination of this extremely immunogenic material, the pentraxins prevent grave auto-immune damage that otherwise would be inflicted on the host's tissues (30). This immunologically silent mode of cellular death supported by the pentraxins might question their opposite activities in defense reactions against extracellular bacteria or fungi. The puzzle of how the pentraxins execute inflammatory anti-microbial responses, while at the same time silencing clonal expansion of autoreactive lymphocytes, has been resolved by new data supplied by Rovere and Baruah with colleagues. These data confirm a dual role for pentraxin 3 in reactions of innate immunity $(35,4)$.

\section{Cross-presentation of exogenous or endogenous antigens}

Auto-immune reactions present a serious menace anytime the host's cells have been damaged up to the point of setting free their intracellular components. A key event in this process is the cross-presentation by DCs of antigenic neoepitopes, originating from the host's tissues, to cytotoxic CD8+ T-lymphocytes (33). This is a common situation in any major surgery, either cardiac or non-cardiac. In the process of cross-presentation, antigens from the extracellular milieu are presented by APCs (antigen-presenting cells) on MHC (major histocompatibility complex) class I molecules in order to stimulate effector CD8+ T lymphocytes. At the same time, cross-presentation is an important mechanism by which the immune system monitors the host's tissues for the presence of foreign (e.g. microbial) antigens or his or her own autoantigens, which are identified by the immune system as foreign or non-self. The outcome of cross-presentation is either immune response or immune tolerance. Which of the two will take place depends on whether isolated antigens are acquired by DCs, leading to tolerance, or whether antigens are acquired along with immunostimulatory signals, leading to immune responses. For dendritic cells, the boundary between an endogenous and an exogenous antigen is not entirely clear-cut. MHC class I-restricted presentation of antigens of both sources is a common situation. From among the plethora of candidate antigens, cross- presentation of those of viral or of the host's own cellular origin numbers among the most common. From the surgical point of view, the latter antigens are of extraordinary importance (32). Cross-presentation of the host's own cellular antigens, which results in cross-tolerance or self-tolerance, sets off deletion of auto-reactive cytotoxic CD8+ T-lymphocytes $(15,6,10)$. Since DCs have been shown to participate both in antigenic cross-presentation and in cross-tolerance, it is no surprise that the same dendritic cell subset is able to contribute both to immunity and to tolerance. Supplementary factors are thus supposed to make the difference between the outcome, i.e. immunity or 
tolerance. A plausible explanation suggests that DCs preferentially induce tolerance to auto-antigens unless exposed to a predominance of inflammatory stimuli, most often those evoked by infection.

\section{Pentraxin 3 in antigenic cross-presentation}

Antigenic cross-presentation is manifold augmented in a milieu overflowed with extracellular antigens, including auto-antigens. Such a situation occurs regularly in areas of tissue injury with multiple and/or deep surgical incisions, often menaced by bacterial invasion and soft tissue trauma/contusion, which may be accompanied by multiple or repeated ischemia-reperfusion processes in wound areas. Extensive cell death sets up an important challenge for the host's immune system. (27). Consequently, pentraxin 3 is recruited at the interface between the host's own dying cells and his or her dendritic cells, which make up efficient cellular sentinels incessantly screening the adjacent environment for the presence foreign antigens. Any time such an antigen has been detected, DCs start off an antigen-specific, MHC-restricted immune response, which is aimed at elimination of intracellular microorganisms, tumor cells or neo/autoantigen-stained self cells or fractions thereof (1). Dendritic cells cross-present antigenic epitopes to cytotoxic CD8+ T-lymphocytes in association with MHC class I molecules, irrespective of the antigenic origin - be it foreign microbial antigens or neo/autoantigen-expressing fragments from the host's own cells. Effector CD8+ T-lymphocytes interact with cross-presented antigens only after the latter have been processed into short peptides by antigenpresenting cells and coupled to MHC class I molecules. Thereafter, cytotoxic CD8+ T lymphocytes identify antigenMHC class I complexes which are designated for elimination (18). Any time antigens of microbial origin and those originating from the host's own cells are presented by DCs to CD8+ T-lymphocytes jointly, a situation commonly encountered in surgical patients, pentraxin 3 cuts up like a useful adaptor of dendritic cell function.

\section{Dual role of pentraxin 3 in auto-immune reactions}

In normal homeostatic conditions, the host's cells dying of apoptotic death start off the production of pentraxin 3 by dendritic cells. Apoptotic cells of the host, to which pentraxin 3 is bound, successfully avoid internalization by immature dendritic cells. The same applies to intracellular processing of apoptotic cells' antigenic load and the crosspresentation of antigen-MHC class I molecule complexes to cytotoxic CD8+ T- lymphocytes. Pentraxin 3 additionally limits the production of pro-inflammatory cytokines and costimulatory molecules, both of which are needed for maturation and the antigen-presenting capacity of DCs (3). In parallel, clonal expansion of T-lymphocytes, which are endowed with the potential to set off cytotoxic auto-immune reactions, is minimized or totally stopped. All the same, it may occur that the host's PTX3-labeled apoptotic cells, due to actual overflow of antigens, notably those of microbial origin, would miss the opportunity to escape ingestion by dendritic cells. If such be the case, pentraxin 3 will enhance the production of proinflammatory cytokines needed to combat infection, while at the same time it will restrict cross-presentation of antigenic neoepitopes to autoreactive CD8+ T-lymphocytes. In so doing, pentraxin 3 will favor defense of the host while at the same time avoiding any undue damage of his or her own tissues whenever microbial infection and extensive cell death coexist. Surgical wounds would be efficiently protected by pentraxin 3 both from infection and from auto-immune damage. On the other hand, pentraxin 3 does not interfere with the presentation to effector cells of soluble antigens, an event required for the elimination of extracellular microbial pathogens. The classical short pentraxin CRP does not substantially contribute to such fine-tuned processes, apparently due to the time lag between the noxious onset and CRP hepatic production, even if the latter is seemingly rapid in execution and undoubtedly robust in volume, with plasma or serum CRP levels reaching several hundredfolds of their basal levels. Nevertheless, if properly regulated, CRP contributes substantially to the generation of an overall anti-inflammatory milieu in which apoptotic cells are cleared by macrophages. CRP-initiated activation of the complement cascade will halt at the level of opsonins $\mathrm{C} 3 \mathrm{~b} / \mathrm{iC} 3 \mathrm{~b}$, thus favoring efficient phagocytosis of opsonin-covered particles. On the other hand, continuation of complement activation up to the generation of the terminal complexes C5b-9(n), which are equipped with mighty cytotoxic and proinflammatory properties, is stopped under these conditions (17).

\section{Conclusion}

Pentraxin 3 is a novel acute phase reactant, whose importance in mammalian biology, including human, is only starting to be recognized. In the setting of acute myocardial infarction and other critical care patients, it may provide a prognostic marker undoubtedly superior in prognostic value to $\mathrm{C}$-reactive protein. At the same time, pentraxin 3 plays a non-redundant role in the humoral arm of innate immunity, protecting the host from some invading microorganisms while at the same time fine-tuning the clearance of apoptotic cells, with deletion of auto-reactive T-lymphocyte clones as a result. The importance of pentraxin 3 in human biology may stand out whenever foreign antigens with those of self-origin are presented jointly, as shown in surgical patients.

\section{Acknowledgements}

This work has been supported by a research program of The Czech Ministry of Youth, Scholarship and Physical Activities No. MSM 0021620812. 


\section{References}

1. Ackerman AL, Cresswell P. Cellular mechanisms governing cross-presentation of exogenous antigens. Nat Immunol 2004;5:678-84.

2. Aprahamian T, Rifkin I, Bonegio R, Hugel B, Freyssinet J-M, Sato K, et al Impaired clearance of apoptotic cells promotes synergy between atherosclerosis and autoimmune disease. J Exp Med 2004;199:1121-31.

3. Baruah P, Dumitriu IE, Peri G, Russo V, Mantovani A, Manfredi AA, RovereQuerini P. The tissue pentraxin PTX3 limits C1q-mediated complement activation and phagocytosis of apoptotic cells by dendritic cells. J Leukocyte Biol 2006;80:87-95.

4. Baruah P, Propato A, Dumitriu IE, Rovere-Querini P, Russo V, Fontana R, et al The pattern recognition receptor PTX3 is recruited at the synapse between dying and dendritic cells and edits the cross-presentation of self, viral and tumor antigens Blood 2006;107:151-8

5. Baumann H, Gauldie J. The acute phase response. Immunol Today 1994; 15 ; $74-80$.

6. Belz GT, Behrens GMN, Smith CM, Miller JFAP, Jones C, Lejon K, et al. The CD8alpha $(+)$ dendritic cell is responsible for inducing peripheral self-tolerance to tissue-associated antigens. J Exp Med 2002;196:1099-104.

7. Bottazzi B, Vouret-Craviari V, Bastone A, De Gioia L, Matteucci C, Peri G, et al. Multimer formation and ligand recognition by the long pentraxin PTX3-similarities and differences with the short pentraxins C-reactive protein and serum amyloid P component. J Biol Chem 1997;272:32817-23.

8. Bozza S, Bistoni F, Gaziano R, Pitzurra L, Zelante T, Bonifazi P, et al. Pentraxin 3 protects from MCMV infection and reactivation through TLR sensing pathways leading to IRF3 activation. Blood First Edition Paper, DOI 10.1182/blood-200603-009266.

9. Breviario F, d'Aniello EM, Golay J, Peri G, Bottazzi B, Bairoch A, et al. Interleukin-1-inducible genes in endothelial cells. Cloning of a new gene related to C-reactive protein and serum amyloid P component. J Biol Chem 1992; 267:22190-7.

10. Davey GM, Kurts C, Miller JFAP, Bouillet P, Strasser A, Brooks AG, Carbone FR, Heath WR. Peripheral deletion of autoreactive CD8 T cells by cross presentation of self-antigen occurs by a Bcl-2-inhibitable pathway mediated by Bim. J Exp Med 2002;196: 947-55.

11. Diniz SN, Nomizo R, Cisalpino PS, Teixeira MM, Brown GD, Mantovani A, et al. PTX3 function as an opsonin for the dectin-1-dependent internalization of zymosan by macrophages J Leukocyte Biol 2004;75:649-56.

12. Doni A, Michela M, Bottazzi B, Peri G, Valentino S, Polentarutti N, Garlanda C, Mantovani A. Regulation of PTX3, a key component of humoral innate immunity in human dendritic cells: stimulation by IL-10 and inhibition by IFN- $\gamma$. J Leukocyte Biol 2006;79:797-802.

13. Fadok VA, Bratton DL, Konowal A, Freed PW, Westcott JY, Henson PM. Macrophages that have ingested apoptotic cells in vitro inhibit proinflammatory cytokine production through autocrine/paracrine mechanisms involving TGF- $\beta$, PGE2, and PAF. J Clin Invest 1998;101:890-8.

14. Fazzini F, Peri G, Doni A, Dell'Antonio G, Dal Cin E, Bozzolo E, et al. PTX3 in small-vessel vasculitides: an independent indicator of disease activity produced at sites of inflammation. Arthritis Rheum 2001;44:2841-50.

15. Ferguson TA, Herndon J, Elzey B, Griffith TS, Schoenberger S, Green DR Uptake of apoptotic antigen-coupled cells by lymphoid dendritic cells and crosspriming of CD8(+) T cells produce active immune unresponsiveness. J Immunol 2002;168:5589-95.

16. Garlanda C, Hirsch E, Bozza S, Salustri M, De Acetis R, Nota A, et al. Non-redundant role of the long pentraxin PTX3 in anti-fungal innate immune response. Nature 2002;420:182-6

17. Gershov D, Kim SJ, Brot N, Elkon KB. C-reactive protein binds to apoptotic cells, protects the cells from assembly of the terminal complement components, and sustains an anti-inflammatory innate immune response: implications for systemic autoimmunity. J Exp Med 2000;192:1353-64
18. Heath WR, Belz GT, Behrens GMN, Smith CM, Forehan SP, Parish IA, et al. Cross-presentation, dendritic cell subsets, and the generation of immunity to cellular antigens. Immunol Rev 2004;199:9-26.

19. Introna M, Vidal Alles V, Castellano M, Picardi G, De Gioia L, Bottazzi B, et al. Cloning of mouse PTX3, a new member of the pentraxin gene family expressed at extrahepatic sites. Blood 1996;87:1862-72.

20. Jeannin P, Bottazzi B, Sironi M, Doni A, Rusnati M, Presta M, et al. Complexity and complementarity of outer membrane protein A recognition by cellular and humoral innate immunity receptors. Immunity 2005;22:551-60.

21. Jeannin P, Renno T, Goetsch L, Miconnet I, Aubry JP, Delneste Y, et al. OmpA targets dendritic cells, induces their maturation and delivers antigen into the MHC class I presentation pathway. Nat Immunol 2000;1:502-9.

22. Klouche M, Brockmeyer N, Knabbe C, Rose-John S. Human herpesvirus 8-derived viral IL-6 induces PTX3 expression in Kaposi's sarcoma cells. AIDS 2002;16:F9-F18

23. Klouche M, Peri G, Knabbe C, Eckstein HH, Schmid FX, Schmitz G, Mantovan A. Modified atherogenic lipoproteins induce expression of pentraxin- 3 by human vascular smooth muscle cells, Atherosclerosis 2004;175:221-8.

24. Latini R, Maggioni AP, Peri G, Gonzini L, Lucci D, Mocarelli P, et al., on behalf of the Lipid Assessment Trial Italian Network (LATIN) Investigators. Prognostic significance of the long pentraxin PTX3 in acute myocardial infarction. Circulation 2004; 110:2349-54

25. Lee GW, Lee TH, Vilček J. TSG-14, a tumor necrosis factor- and IL-1-inducible protein, is a novel member of the pentraxin family of acute phase proteins. J Immunol 1993;150:1804-12.

26. Malaguarnera L, Pilastro MR, Vicari L, Di Marco R, Malaguarnera M, Messina A. PTX3 gene expression in Castleman's disease. Eur J Haematol 2000;64:132-4. 27. Morelli AE, Thomson AW. Dendritic cells: regulators of alloimmunity and opportunities for tolerance induction. Immunol. Rev 2003;196:125-46.

28. Napoleone E, Di Santo A, Bastone A, Peri G, Mantovani A, de Gaetano G, et al. Long pentraxin PTX3 upregulates tissue factor expression in human endothelial cells: a novel link between vascular inflammation and clotting activation. Arterioscler Thromb Vasc Biol 2002;22:782-7.

29. Nauta AJ, Bottazzi B, Mantovani A, Salvatori G, Kishore U, Schwaeble WJ, et al. Biochemical and functional characterization of the interaction between pentraxin 3 and C1q. Eur J Immunol 2003;33:465-73.

30. Nauta AJ, Daha MR, van Kooten C, Roos A. Recognition and clearance of apoptotic cells: a role for complement and pentraxins. Trends Immunol 2003; 24:148-54.

31. Peri G, Introna M, Corradi D, Iacuitti G, Signorini S, Avanzini F, et al. PTX3, a prototypical long pentraxin, is an early indicator of acute myocardial infarction in humans. Circulation 2000;102:636-41.

32. Ramirez MC, Sigal LJ. Macrophages and dendritic cells use the cytosolic pathway to rapidly cross-present antigen from live, vaccinia-infected cells. J Immunol 2002;169: 6733-42.

33. Rock KL, Shen L. Cross-presentation: underlying mechanisms and role in immune surveillance. Immunol Rev 2005;207:166-83

34. Rolph MS, Zimmer S, Bottazzi B, Garlanda C, Mantovani A, Hansson GK. Production of the long pentraxin PTX3 in advanced atherosclerotic plaques. Arterioscler Thromb Vasc Biol 2002;22:e10-e14.

35. Rovere P, Peri G, Fazzini F, Bottazzi B, Doni A, Bondanza A, et al. The long pentraxin PTX3 binds to apoptotic cells and regulates their clearance by antigen-presenting dendritic cells. Blood 2000;96:4300-6.

36. Rovere-Querini P, Dumitriu IE. Corpse disposal after apoptosis. Apoptosis 2003;8:469-79

37. Vouret-Craviari V, Matteucci C, Peri G, Poli G, Introna M, Mantovani A Expression of a long pentraxin, PTX3, by monocytes exposed to the mycobacterial cell wall component lipoarabinomannan. Infect Immun 1997;65: 1345-50.

38. Wisniewski H-G, Vilček J. Cytokine-induced gene expression at the crossroads of innate immunity, inflammation and fertility: TSG-6 and PTX3/TSG-14. Cytokine Growth Factor Rev 2004;15:129-46.

\section{Corresponding author:}

Pavel Kuneš, M.D., University Hospital in Hradec Králové, Department of Cardiac Surgery Sokolská 581, 50005 Hradec Králové, Czech Republic, e-mail: kunes.pavel@fnhk.cz 